\title{
Regularity Criterion for the Nematic Liquid Crystal Flows in Terms of Velocity
}

\author{
Ruiying Wei, ${ }^{1,2}$ Zheng-an Yao, ${ }^{1}$ and $\mathrm{Yin}_{\mathrm{Li}}{ }^{1,2}$ \\ ${ }^{1}$ Department of Mathematics and Computational Science, Sun Yat-Sen University, Guangzhou 510275, China \\ ${ }^{2}$ School of Mathematics and Information Science, Shaoguan University, Shaoguan 512005, China \\ Correspondence should be addressed to Ruiying Wei; weiruiying521@163.com
}

Received 7 February 2014; Accepted 15 May 2014; Published 30 June 2014

Academic Editor: Gaohang Yu

Copyright (c) 2014 Ruiying Wei et al. This is an open access article distributed under the Creative Commons Attribution License, which permits unrestricted use, distribution, and reproduction in any medium, provided the original work is properly cited.

We study the regularity criterion for the 3D nematic liquid crystal flows in the framework of anisotropic Lebesgue space. More precisely, we proved some sufficient conditions in terms of velocity or the fractional derivative of velocity in one direction.

\section{Introduction}

This paper is devoted to the regularity criterion for the threedimensional nematic liquid crystal flows:

$$
\begin{gathered}
u_{t}+(u \cdot \nabla) u+\nabla p-v \Delta u=-\lambda \nabla \cdot(\nabla d \odot \nabla d), \\
x \in R^{3}, \quad t>0, \\
d_{t}+(u \cdot \nabla) d=\gamma(\Delta d-f(d)), \quad x \in R^{3}, t>0, \\
\nabla \cdot u=0, \quad x \in R^{3}, t>0,
\end{gathered}
$$

with initial data

$$
\left.(u, d)\right|_{t=0}=\left(u_{0}, d_{0}\right), \quad x \in R^{3},
$$

where $u(x, t)$ is the velocity field, $d(x, t)$ represents the macroscopic average of the nematic liquid crystal orientation field, and $p(x, t)$ is the scalar pressure. The symbol $\nabla d \odot \nabla d$ denotes a matrix whose $(i, j)$ th entry is given by $\partial_{i} d \cdot \partial_{j} d$ for $1 \leq i, j \leq 3$; here $f(d)=\left(1 / \varepsilon^{2}\right)\left(|d|^{2}-1\right) d$. Since the sizes of the viscosity constants $v, \lambda, \gamma, \varepsilon$ do not play important roles in our proof, for simplicity, we assume all these positive constants to be one.

The hydrodynamic theory of liquid crystals was established by Ericksen and Leslie [1-4]; the model (1) is a simplified system of Ericksen-Leslie model which was first introduced by Lin in [5], and one of the most significant works is given by Lin and Liu [6]; more precisely, they established global existence for weak solutions and classical solutions. Recently, Liu et al. in [7] established the regularity criterion for (1) as follows:

$$
\int_{0}^{T}\left\|\partial_{3} u(\tau)\right\|_{L^{\alpha}}^{\beta} d \tau<\infty, \quad \text { with } \frac{2}{\beta}+\frac{3}{\alpha} \leq 1, \alpha>3 .
$$

One may refer to some interesting and important regularity criteria of nematic liquid crystal flows studied by many authors (see, e.g., [8-13] and the references therein). When $d$ is constant, the system (1) becomes the well-known NavierStokes equations. The regularity of solutions to the 3D NS equations has been widely investigated during the past fifty years; see, for example, [14-22] and so on. The aim of this paper is to establish a new regularity criterion by providing sufficient condition in terms of velocity or the fractional derivative of velocity in one direction in the framework of anisotropic Lebesgue space.

Throughout the paper, the norm of the Lebesgue spaces $L^{p}\left(R^{3}\right)$ is denoted by $\|\cdot\|_{L^{p}}$ and denoted the directional derivatives of a function $\phi$ by $\partial_{i} \phi=\left(\partial \phi / \partial x_{i}\right)(i=1,2,3)$, the symbol $\int f(x) d x=\int_{R^{3}} f(x) d x, \Lambda_{i}=\sqrt{-\partial_{i}^{2}}, \Lambda=\sqrt{-\Delta}$, 
\|\|$\phi\left\|_{L_{i}^{p}}\right\|_{L_{j, k}^{q}}=\left(\int_{R^{2}}\left(\int_{R}|\phi(x)|^{p} d x_{i}\right)^{q / p} d x_{j} d x_{k}\right)^{1 / q}$, and $(i, j, k)$ belongs to the permutation group $S=\operatorname{span}\{1,2,3\}$. Denote

$$
\begin{aligned}
& E_{1}=\left\{(\gamma, \alpha) \in(2, \infty]^{2}, \frac{1}{\gamma}+\frac{2}{\alpha}<1\right\}, \\
& E_{2}=\left\{\alpha \in(2, \infty], \frac{3-\alpha r}{\alpha}<1, \frac{1-\alpha r}{\alpha-2}<1\right\} .
\end{aligned}
$$

Theorem 1. Let $\left(u_{0}, d_{0}\right) \in H^{1}\left(R^{3}\right) \times H^{2}\left(R^{3}\right)$ with the initial data div $u_{0}=0$, and let the pair $(u, b)$ be the weak solution to the liquid crystal flows (1)-(2) on $[0, T)$ for some $0<T<\infty$. If $u$ satisfies

$$
\begin{array}{r}
\int_{0}^{T}\|\| u(\tau)\left\|_{L_{i}^{\gamma}}\right\|_{L_{j, k}^{\alpha}}^{\beta} d \tau<\infty, \text { with } \frac{2}{\beta}+\frac{2}{\alpha}+\frac{1}{\gamma} \leq 1, \\
(\gamma, \alpha) \in E_{1}
\end{array}
$$

then $(u, d)$ can be extended beyond $T$.

Theorem 2. Let $\left(u_{0}, d_{0}\right) \in H^{1}\left(R^{3}\right) \times H^{2}\left(R^{3}\right)$ with the initial data div $u_{0}=0$, and let the pair $(u, b)$ be the weak solution to the liquid crystal flows (1)-(2) on $[0, T)$ for some $0<T<\infty$. If $u$ satisfies

$$
\begin{aligned}
& \int_{0}^{T}\left\|\Lambda_{i}^{r} u(\tau)\right\|_{L^{\alpha}}^{\beta} d \tau<\infty \text {, with } \\
& \frac{2}{\beta}+\frac{3}{\alpha} \leq \begin{cases}1+\frac{1}{\alpha}, \quad \alpha \in(2, \infty), & \text { if } r \in\left(\frac{1}{\alpha}, 1\right], \\
\text { or } & \text { if } r \in\left[0, \frac{1}{\alpha}\right),\end{cases}
\end{aligned}
$$

then $(u, d)$ can be extended beyond $T$.

Corollary 3. Under the assumption of Theorem 2, if we fix $r=1$, then the sufficient condition is that

$$
\begin{array}{r}
\int_{0}^{T}\left\|\partial_{i} u(\tau)\right\|_{L^{\alpha}}^{\beta} d \tau<\infty, \quad \text { with } \frac{2}{\beta}+\frac{3}{\alpha} \leq 1+\frac{1}{\alpha}, \\
\alpha \in(2, \infty) .
\end{array}
$$

Remark 4. Comparing with the corresponding results in [7], it is obvious that the conclusion of Corollary 3 is an improvement version of Theorem 1.1 in [7] in some sense.

\section{The Proof of Theorems 1 and 2}

In this section, we will prove Theorems 1 and 2. For convenience, we first recall the following three-dimensional Sobolev and Ladyzhenskaya inequalities in the whole space (see, e.g., [23-25]).
Lemma 5. Let $2 \leq q \leq 6,2 \leq p<\infty$, and $2<r, s \leq \infty$. There hold that

$$
\begin{aligned}
& \|\phi\|_{L^{q}} \leq C\|\phi\|_{L^{2}}^{(6-q) / 2 q}\left\|\partial_{1} \phi\right\|_{L^{2}}^{(q-2) / 2 q}\left\|\partial_{2} \phi\right\|_{L^{2}}^{(q-2) / 2 q}\left\|\partial_{3} \phi\right\|_{L^{2}}^{(q-2) / 2 q}, \\
& \|\| \phi\left\|_{L_{i, j}^{p}}\right\|_{L_{k}^{2}} \leq C\|\phi\|_{L^{2}}^{2 / p}\left\|\partial_{i} \phi\right\|_{L^{2}}^{(p-2) / 2 p}\left\|\partial_{j} \phi\right\|_{L^{2}}^{(p-2) / 2 p}, \\
& \|\| \phi\left\|_{L_{i}^{2 r /(r-2)}}\right\|_{L_{j, k}^{2 s /(s-2)}} \leq C\|\phi\|_{L^{2}}^{1-(1 / r)-(2 / s)}\left\|\partial_{i} \phi\right\|_{L^{2}}^{1 / r}\left\|\partial_{j} \phi\right\|_{L^{2}}^{1 / s}\left\|\partial_{k} \phi\right\|_{L^{2}}^{1 / s} .
\end{aligned}
$$

Proof of Theorem 1. Suppose that $\left[0, T^{*}\right)$ is the maximal interval of the existence of the local smooth solution. If $T^{*} \geq T$, then there is nothing to prove; on the other side, for $T^{*}<T$, our strategy is to show that

$$
\limsup _{t \rightarrow T^{*}}\left(\|\nabla u(\cdot, t)\|_{L^{2}}^{2}+\|\Delta d(\cdot, t)\|_{L^{2}}^{2}\right) \leq C,
$$

under the assumption (5). As a result, the interval $\left[0, T^{*}\right.$ ) cannot be a maximal interval of existence, which leads to a contradiction.

We multiply (1) by $u$ and integrate over $R^{3}$ and, similarly, multiply (1) $)_{2}$ by $-\Delta d+f(d)$ and integrate over $R^{3}$ and then by adding two results above and using the fact that $\nabla \cdot(\nabla d \odot \nabla d)=$ $\nabla\left(|\nabla d|^{2} / 2\right)+\Delta d \nabla d$, we obtain

$$
\begin{aligned}
\frac{1}{2} \frac{d}{d t} \int & \left(|u|^{2}+|\nabla d|^{2}+\frac{1}{2}\left(|d|^{2}-1\right)^{2}\right) d x \\
& +\int\left(|\nabla u|^{2}+|\Delta d-f(d)|^{2}\right) d x=0 .
\end{aligned}
$$

Here we used the facts that $\operatorname{div} u=0$ and $(u \cdot \nabla u, u)=$ $(u, \nabla p)=(u \cdot \nabla d, f(d))=\left(u, \nabla\left(|d|^{2} / 2\right)\right)=0$; here $(\cdot, \cdot)$ denotes the usual inner product of $L^{2}\left(R^{3}\right)$, which implies

$$
\|u\|_{L^{\infty}\left(0, T ; L^{2}\right)}+\|u\|_{L^{2}\left(0, T ; H^{1}\right)} \leq C .
$$

Besides, we multiply $(1)_{2}$ by $|d|^{4} d$ and integrate over $R^{3}$ and get

$$
\frac{1}{6} \frac{d}{d t} \int|u|^{6} d x+\int\left(5|d|^{4}|\nabla d|^{2}+|d|^{8}\right) d x=\int|d|^{6} d x
$$

which implies

$$
\|d(\cdot, t)\|_{L^{\infty}\left(0, T ; L^{6}\right)} \leq C\left\|d_{0}\right\|_{L^{6}} \leq C\left\|d_{0}\right\|_{H^{1}} .
$$

Multiplying the first equation of (1) by $-\Delta u$ and integrating over $R^{3}$. Similarly, by taking $\Delta$ on both sides of the second equation of (1), by multiplying the resulting equation by $\Delta d$, by integrating over $R^{3}$, and then by adding two results above and taking the divergence-free condition $\operatorname{div} u=0$ into account, we obtain

$$
\begin{aligned}
& \frac{1}{2} \frac{d}{d t}\left(\|\nabla u\|_{L^{2}}^{2}+\|\Delta d\|_{L^{2}}^{2}\right)+\|\Delta u\|_{L^{2}}^{2}+\|\nabla \Delta d\|_{L^{2}}^{2} \\
& =\int(u \cdot \nabla) u \cdot \Delta u d x \\
& \quad-2 \sum_{i=1}^{3} \int \nabla u_{i} \partial_{i} \nabla d \Delta d d x-\int \Delta f(d) \Delta d d x \\
& =I_{1}+I_{2}+I_{3} .
\end{aligned}
$$


In the following, we establish the bounds of $I_{1}-I_{3}$, for the first term $I_{1}$; thanks to Lemma 5 and using Young's inequality, we have

$$
\begin{aligned}
I_{1} \leq & \int|u||\nabla u||\Delta u| d x \\
\leq & \|\| u\left\|_{L_{3}^{\nu}}\right\|_{L_{1,2}^{\alpha}}\|\| \nabla u\left\|_{L_{3}^{2 \gamma /(\gamma-2)}}\right\|_{L_{1,2}^{2 \alpha /(\alpha-2)}}\|\Delta u\|_{L^{2}} \\
\leq & C\|\| u\left\|_{L_{3}^{\gamma}}\right\|_{L_{1,2}^{\alpha}}\|\nabla u\|_{L^{2}}^{1-(1 / \gamma)-(2 / \alpha)} \\
& \times\left\|\partial_{1} \nabla u\right\|_{L^{2}}^{1 / \alpha}\left\|\partial_{2} \nabla u\right\|_{L^{2}}^{1 / \alpha}\left\|\partial_{3} \nabla u\right\|_{L^{2}}^{1 / \gamma}\|\Delta u\|_{L^{2}} \\
\leq & C\|\| u\left\|_{L_{3}^{\gamma}}\right\|_{L_{1,2}^{\alpha}}\|\nabla u\|_{L^{2}}^{1-(1 / \gamma)-(2 / \alpha)}\|\Delta u\|_{L^{2}}^{(1 / \gamma)+(2 / \alpha)+1} \\
\leq & \frac{1}{2}\|\Delta u\|_{L^{2}}^{2}+C\|\| u\left\|_{L_{3}^{\gamma}}\right\|_{L_{1,2}^{\alpha}}^{2 \alpha \gamma /(\alpha \gamma-2 \gamma-\alpha)}\|\nabla u\|_{L^{2}}^{2} .
\end{aligned}
$$

For the second term $I_{2}$, similar to estimate of $I_{1}$, we have

$$
\begin{aligned}
I_{2}= & -2 \sum_{i=1}^{3} \int \nabla u_{i} \partial_{i} \nabla d \Delta d d x \\
= & 2 \sum_{i, j=1}^{3} \int\left(u_{i} \partial_{j} \partial_{i} \partial_{j} d \Delta d d x+u_{i} \partial_{i} \partial_{j} d \partial_{j} \Delta d\right) d x \\
\leq & C \int|u|\left|\nabla^{2} d\right||\nabla \Delta d| d x \\
\leq & C\|\| u\left\|_{L_{3}^{\gamma}}\right\|_{L_{1,2}^{\alpha}}\|\Delta d\|_{L^{2}}^{1-(1 / \gamma)-(2 / \alpha)} \\
& \times\left\|\partial_{1} \Delta d\right\|_{L^{2}}^{1 / \alpha}\left\|\partial_{2} \Delta d\right\|_{L^{2}}^{1 / \alpha}\left\|\partial_{3} \Delta d\right\|_{L^{2}}^{1 / \gamma}\|\nabla \Delta d\|_{L^{2}} \\
\leq & \frac{1}{4}\|\nabla \Delta d\|_{L^{2}}^{2}+C\|\| u\left\|_{L_{3}^{\gamma}}\right\|_{L_{1,2}^{\alpha}}^{2 \alpha /(\alpha \gamma-2 \gamma-\alpha)}\|\Delta d\|_{L^{2}}^{2} .
\end{aligned}
$$

For the term $I_{3}$, using Hölder's inequality, Young's inequality, and (13), one has

$$
\begin{aligned}
I_{3} & =-\int \Delta f(d) \Delta d d x=\int \nabla\left(|d|^{2} d\right) \cdot \nabla \Delta d d x+\|\Delta d\|_{L^{2}}^{2} \\
& =3 \int|d|^{2} \nabla d \cdot \nabla \Delta d d x+\|\Delta d\|_{L^{2}}^{2} \\
& \leq C\|d\|_{L^{6}}^{2}\|\nabla d\|_{L^{6}}\|\nabla \Delta d\|_{L^{2}}+\|\Delta d\|_{L^{2}}^{2} \\
& \leq \frac{1}{4}\|\nabla \Delta d\|_{L^{2}}^{2}+C\|\Delta d\|_{L^{2}}^{2} .
\end{aligned}
$$

Substituting the above estimates (15)-(17) into (14), we obtain

$$
\begin{aligned}
& \frac{d}{d t}\left(\|\nabla u\|_{L^{2}}^{2}+\|\Delta d\|_{L^{2}}^{2}\right)+\|\Delta u\|_{L^{2}}^{2}+\|\nabla \Delta d\|_{L^{2}}^{2} \\
& \quad \leq C\left(1+\|\| u\left\|_{L_{3}^{\prime}}\right\|_{L_{1,2}^{\alpha}}^{2 \alpha \gamma /(\alpha \gamma-2 \gamma-\alpha)}\right)\|\nabla u\|_{L^{2}}^{2}+C\|\Delta d\|_{L^{2}}^{2} .
\end{aligned}
$$

Integrating (18) from 0 to $t$, using Hölder's inequality and Young's inequality, one has

$$
\begin{aligned}
& \|\nabla u\|_{L^{2}}^{2}+\|\Delta d\|_{L^{2}}^{2}+\int_{0}^{t}\left(\|\Delta u\|_{L^{2}}^{2}+\|\nabla \Delta d\|_{L^{2}}^{2}\right) d \tau \\
& \leq C \int_{0}^{t}\left(1+\|\| u\left\|_{L_{3}^{\nu}}\right\|_{L_{1,2}^{\alpha}}^{2 \alpha \gamma /(\alpha \gamma-2 \gamma-\alpha)}\right) \\
& \quad \times\left(\|\nabla u\|_{L^{2}}^{2}+\|\Delta d\|_{L^{2}}^{2}\right) d \tau+\left\|\nabla u_{0}\right\|_{L^{2}}^{2}+\left\|\Delta d_{0}\right\|_{L^{2}}^{2} .
\end{aligned}
$$

Finally, applying Gronwall's inequality and using condition (5), then $(u, d)$ can be extended beyond $T$. This completes the proof of Theorem 1.

Proof of Theorem 2. When $r \in[0,1 / \alpha)$, combining Theorem 1 and using the following imbedding theorem, one can get the conclusion that

$$
\|\| u\left\|_{L_{i}^{\alpha /(1-\alpha r)}}\right\|_{L_{j, k}^{\alpha}} \leq C\left\|\Lambda_{i}^{r} u\right\|_{L^{\alpha}} .
$$

When $r \in(1 / \alpha, 1)$, our strategy is to show that

$$
\int_{0}^{t}\left\|\Lambda_{i}^{r} u(\tau)\right\|_{L^{\alpha}}^{\beta} d \tau<\infty, \quad \frac{2}{\beta}+\frac{3}{\alpha} \leq 1+\frac{1}{\alpha}, \quad \alpha \in(2, \infty)
$$

is a sufficient condition. We can verify that integral term $\int_{0}^{t}\|\| u(\tau)\left\|_{L_{i}^{\infty}}\right\|_{L_{j, k}^{\delta}}^{2 \delta /(\delta-2)} d \tau$ satisfies the conditions of Theorem 1 with $\delta \in(2, \infty)$. Applying Lemma 5, Hölder's inequality, and the interpolation theorem, one can conclude that, for $\delta \in$ $[\alpha(2 r+1) / r \alpha, \alpha]$,

$$
\begin{aligned}
\|\| u\left\|_{L_{i}^{\infty}}\right\|_{L_{j, k}^{\delta}} \leq & C\|\| u\left\|_{L_{i}^{2}}^{\theta}\right\| \Lambda_{i}^{r} u\left\|_{L_{i}^{\alpha}}^{1-\theta}\right\|_{L_{j, k}^{\delta}} \\
\leq & C\|\| u\left\|_{L_{i}^{2}}\right\|_{L_{j, k}^{p}}^{\theta}\|\| \Lambda_{i}^{r} u\left\|_{L_{i}^{\alpha}}\right\|_{L_{j, k}^{\alpha}}^{1-\theta} \\
\leq & C\|\| u\left\|_{L_{j, k}^{p}}\right\|_{L_{i}^{2}}^{\theta}\left\|\Lambda_{i}^{r} u\right\|_{L^{\alpha}}^{1-\theta} \\
\leq & C\|u\|_{L^{2}}^{2 \theta / p}\left\|\partial_{j} u\right\|_{L^{2}}^{(p-2) \theta / 2 p} \\
& \times\left\|\partial_{k} u\right\|_{L^{2}}^{(p-2) \theta / 2 p}\left\|\Lambda_{i}^{r} u\right\|_{L^{\alpha}}^{1-\theta} \\
\leq & C\|u\|_{L^{2}}^{2 \theta / p}\|\nabla u\|_{L^{2}}^{(p-2) \theta / p}\left\|\Lambda_{i}^{r} u\right\|_{L^{\alpha}}^{1-\theta},
\end{aligned}
$$

where $1 / \delta=(\theta / p)+((1-\theta) / \alpha)$ with $\theta=2(r \alpha-1) /(2(r \alpha-$ $1)+\alpha)$ and we have used the fact that $\delta \geq((2 r+1) \alpha-2) / \alpha r$ implies $p \geq 2$. Using Hölder's inequality, one has

$$
\begin{aligned}
& \int_{0}^{t}\|\| u(\tau)\left\|_{L_{i}^{\infty}}\right\|_{L_{j, k}^{\delta}}^{2 \delta /(\delta-2)} d \tau \\
& \quad \leq C \int_{0}^{t}\|u\|_{L^{2}}^{4 \delta \theta / p(\delta-2)}\|\nabla u\|_{L^{2}}^{2 \delta \theta(p-2) / p(\delta-2)}
\end{aligned}
$$




$$
\begin{aligned}
& \times\left\|\Lambda_{i}^{r} u\right\|_{L^{\alpha}}^{2(1-\theta) \delta /(\delta-2)} d \tau \\
\leq & C\|u\|_{L_{t}^{\infty} L^{2}}^{4 \delta \theta / p(\delta-2)}\|\nabla u\|_{L_{t}^{2} L^{2}}^{2 \delta \theta(p-2) / p(\delta-2)} \\
& \times\left(\int_{0}^{t}\left\|\Lambda_{i}^{r} u\right\|_{L^{\alpha}}^{(2(1-\theta) \delta /(\delta-2)) \eta} d \tau\right)^{1 / \eta},
\end{aligned}
$$

where $\eta=p(\delta-2) /(p(\delta-2)-\delta \theta(p-2))$.

According to the fact that $1 / \delta=(\theta / p)+((1-\theta) / \alpha)$ and $\theta=2(r \alpha-1) /(2(r \alpha-1)+\alpha)$, we have

$$
\begin{aligned}
& \frac{2(1-\theta) \delta}{\delta-2} \eta \\
& =\frac{2 p \delta(1-\theta)}{p(\delta-2)-\delta \theta(p-2)}=\frac{2 \delta(1-\theta)}{\delta-2-\delta \theta(1-(2 / p))} \\
& =\frac{2 \delta(1-\theta)}{\delta-2-\delta \theta+2 \delta(1 / \delta)-2 \delta((1-\theta) / \alpha)} \\
& =\frac{2 \delta(1-\theta)}{\delta-\delta \theta-(2 \delta(1-\theta) / \alpha)}=\frac{2(1-\theta)}{1-\theta-(2(1-\theta) / \alpha)} \\
& =\frac{2 \alpha(1-\theta)}{\alpha-\alpha \theta-2(1-\theta)}=\frac{2 \alpha}{\alpha-2} .
\end{aligned}
$$

This together with Theorem 1 gives the desired result of Theorem 2.

\section{Conflict of Interests}

The authors declare that there is no conflict of interests regarding the publication of this paper.

\section{Acknowledgments}

This work is partially supported by the NNSFC (Grant no. 11271381), China, 973 Program (Grant no. 2011CB808002), Guangdong Provincial Culture of Seedling of China (no. 2013LYM0081), Guangdong Provincial NSF of China (no. S2012010010069), the Shaoguan Science and Technology Foundation (no. 313140546), and Science Foundation of Shaoguan University.

\section{References}

[1] J. L. Ericksen, "Conservation laws for liquid crystals," Transactions of the Society of Rheology: Journal of Rheology, vol. 5, no. 1, pp. 23-34, 1961.

[2] J. L. Ericksen, "Continuum theory of nematic liquid crystals," Res Mechanica, vol. 21, no. 4, pp. 381-392, 1987.

[3] J. L. Ericksen, "Liquid crystals with variable degree of orientation," Archive for Rational Mechanics and Analysis, vol. 113, no. 2, pp. 97-120, 1990.

[4] F. Leslie, Theory of Flow Phenomenum in Liquid Crystals, vol. 4, Springer, New York, NY, USA, 1979.

[5] F.-H. Lin, "Nonlinear theory of defects in nematic liquid crystals: phase transition and flow phenomena," Communications on Pure and Applied Mathematics, vol. 42, no. 6, pp. 789-814, 1989.
[6] F.-H. Lin and C. Liu, "Nonparabolic dissipative systems modeling the flow of liquid crystals," Communications on Pure and Applied Mathematics, vol. 48, no. 5, pp. 501-537, 1995.

[7] Q. Liu, J. Zhao, and S. Cui, "A regularity criterion for the threedimensional nematic liquid crystal flow in terms of one directional derivative of the velocity," Journal of Mathematical Physics, vol. 52, no. 3, Article ID 033102, 2011.

[8] J. Fan and B. Guo, "Regularity criterion to some liquid crystal models and the Landau-Lifshitz equations in $R^{3}$," Science in China A: Mathematics, vol. 51, no. 10, pp. 1787-1797, 2008.

[9] Q. Liu and J. Zhao, "Logarithmically improved blow-up criteria for the nematic liquid crystal flows," Nonlinear Analysis: Real World Applications, vol. 16, pp. 178-190, 2014.

[10] T. Huang and C. Wang, "Blow up criterion for nematic liquid crystal flows," Communications in Partial Differential Equations, vol. 37, no. 5, pp. 875-884, 2012.

[11] F. Lin, J. Lin, and C. Wang, "Liquid crystal flows in two dimensions," Archive for Rational Mechanics and Analysis, vol. 197, no. 1, pp. 297-336, 2010.

[12] Z. Zhang, X. Wang, and Z.-A. Yao, "Remarks on regularity criteria for the weak solutions of liquid crystals," Journal of Evolution Equations, vol. 12, no. 4, pp. 801-812, 2012.

[13] Z. Zhang, T. Tang, and L. Liu, "An Osgood type regularity criterion for the liquid crystal flows," Nonlinear Differential Equations and Applications, vol. 21, no. 2, pp. 253-262, 2014.

[14] G. P. Galdi, An Introduction to the Mathematical Theory of the Navier-Stokes Equations, vol. 1, Springer, New York, NY, USA, 1994.

[15] G. P. Galdi, An Introduction to the Mathematical Theory of the Navier-Stokes Equations, vol. 2, Springer, New York, NY, USA, 1994.

[16] P. Penel and M. Pokorný, "Some new regularity criteria for the Navier-Stokes equations containing gradient of the velocity," Applications of Mathematics, vol. 49, no. 5, pp. 483-493, 2004.

[17] C. Cao and E. S. Titi, "Regularity criteria for the threedimensional Navier-Stokes equations," Indiana University Mathematics Journal, vol. 57, no. 6, pp. 2643-2661, 2008.

[18] S. Gala, "Remarks on regularity criterion for weak solutions to the Navier-Stokes equations in terms of the gradient of the pressure," Applicable Analysis, vol. 92, no. 1, pp. 96-103, 2013.

[19] X. Jia and Y. Zhou, "Remarks on regularity criteria for the Navier-Stokes equations via one velocity component," Nonlinear Analysis: Real World Applications, vol. 15, pp. 239-245, 2014.

[20] Y. Zhou and M. Pokorný, "On the regularity of the solutions of the Navier-Stokes equations via one velocity component," Nonlinearity, vol. 23, no. 5, pp. 1097-1107, 2010.

[21] Z. Zhang, "A Serrin-type regularity criterion for the NavierStokes equations via one velocity component," Communications on Pure and Applied Analysis, vol. 12, no. 1, pp. 117-124, 2013.

[22] Z. Zhang, Z.-A. Yao, M. Lu, and L. Ni, "Some Serrin-type regularity criteria for weak solutions to the Navier-Stokes equations," Journal of Mathematical Physics, vol. 52, no. 5, Article ID 053103, 2011.

[23] R. A. Adams, Sobolev Spaces, Academic Press, New York, NY, USA, 1975.

[24] X. Zheng, "A regularity criterion for the tridimensional NavierStokes equations in term of one velocity component," Journal of Differential Equations, vol. 256, no. 1, pp. 283-309, 2014.

[25] O. A. Ladyzhenskaya, Mathematical Theory of Viscous Incompressible Flow, Gordon and Breach, New York, NY, USA, 2nd edition, 1969, English translation. 


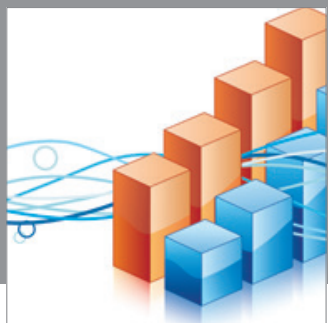

Advances in

Operations Research

mansans

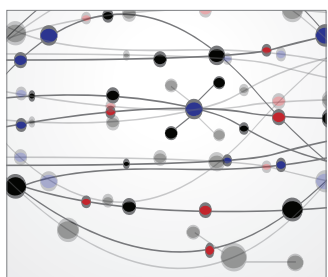

The Scientific World Journal
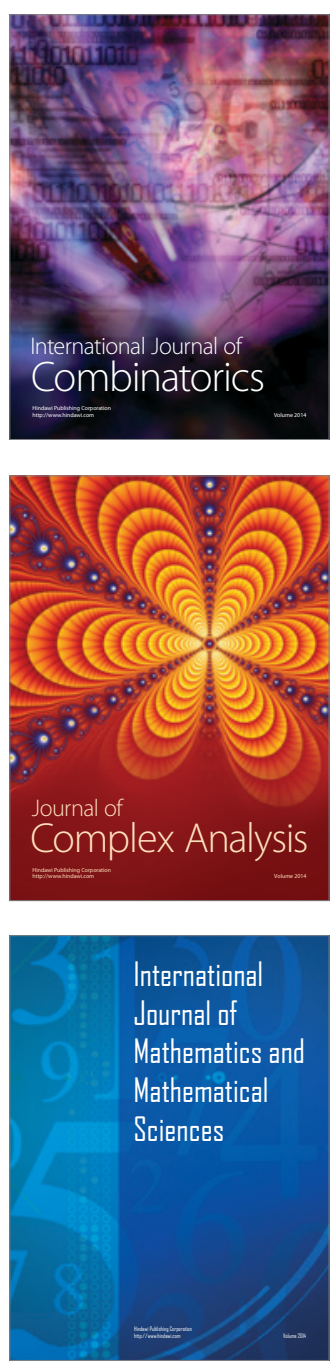
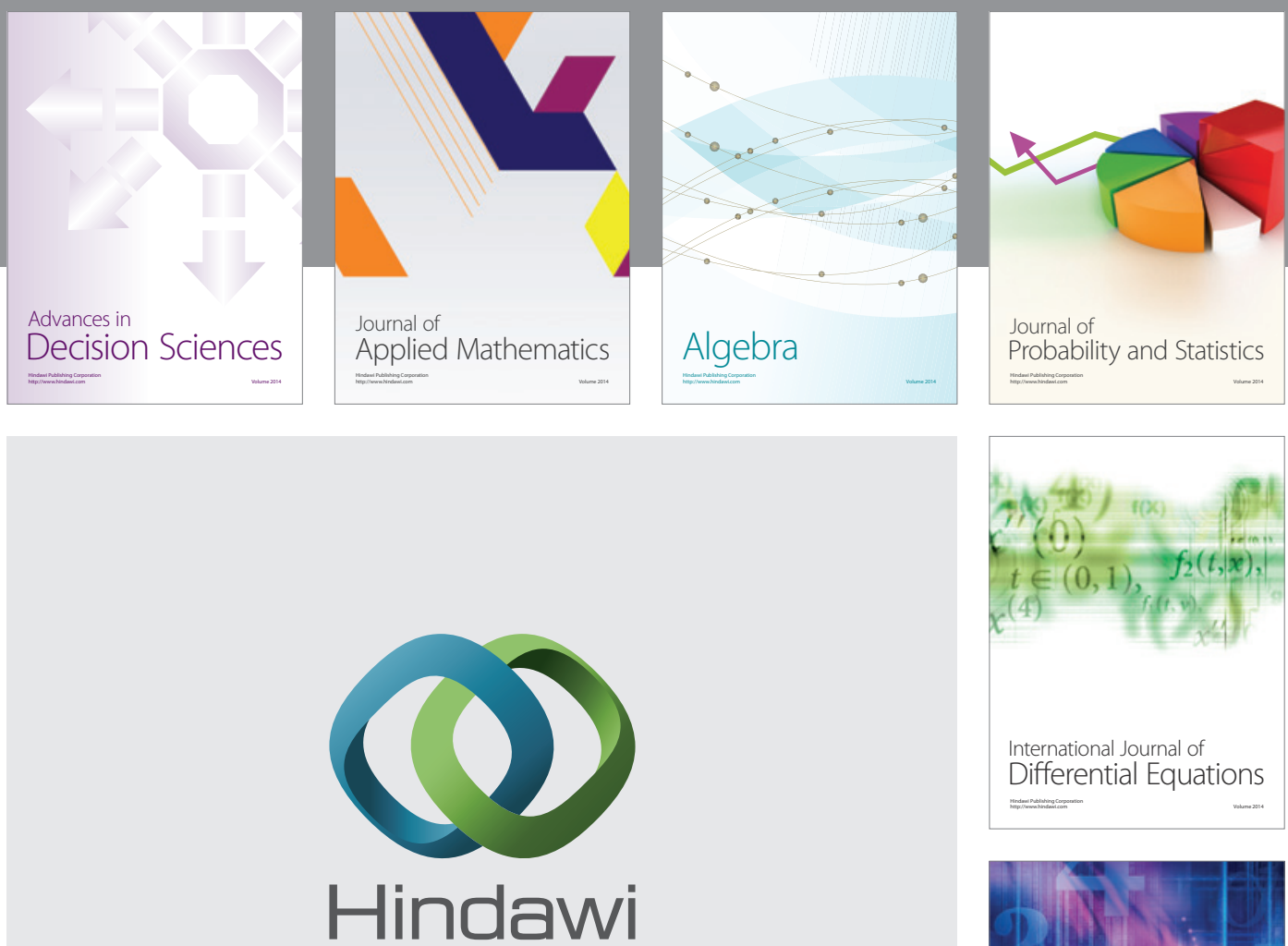

Submit your manuscripts at http://www.hindawi.com
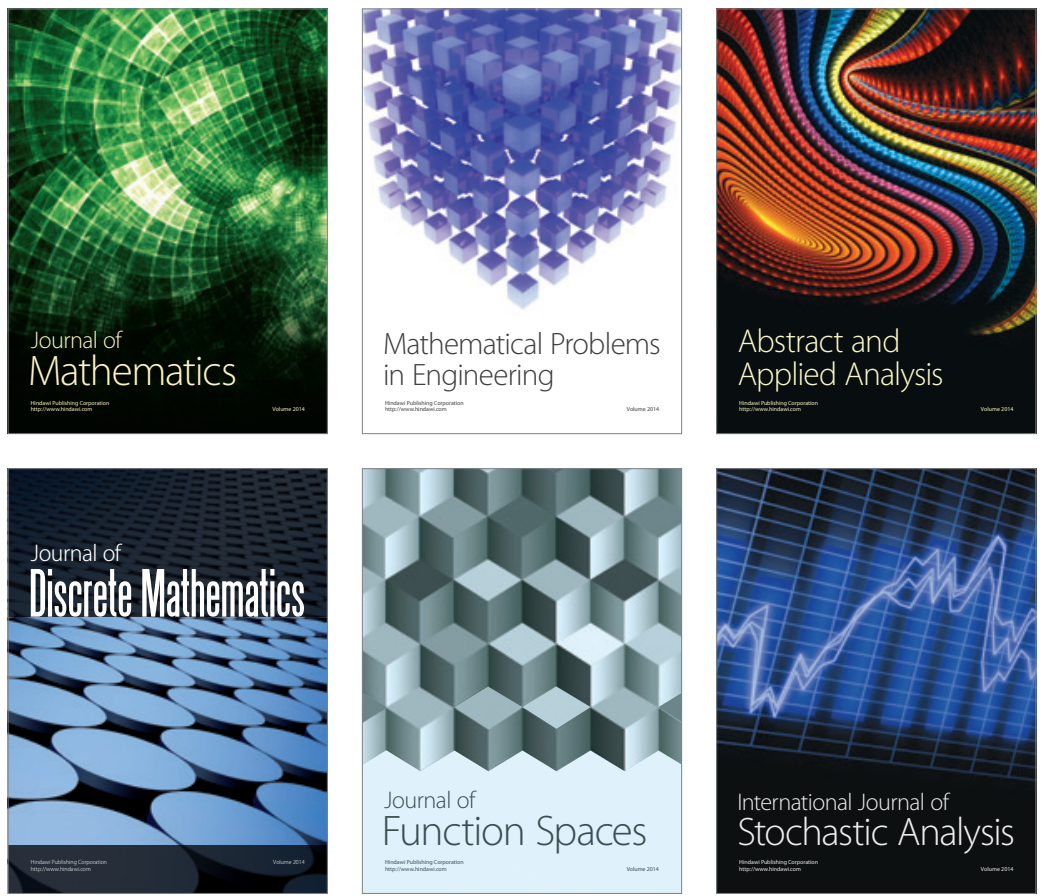

Journal of

Function Spaces

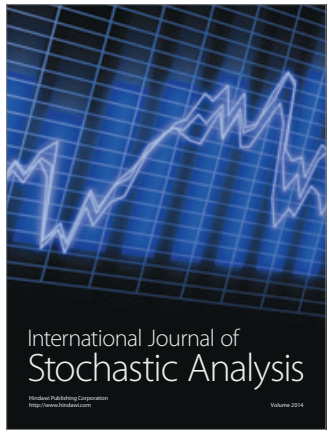

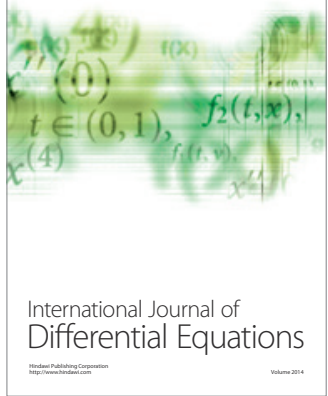
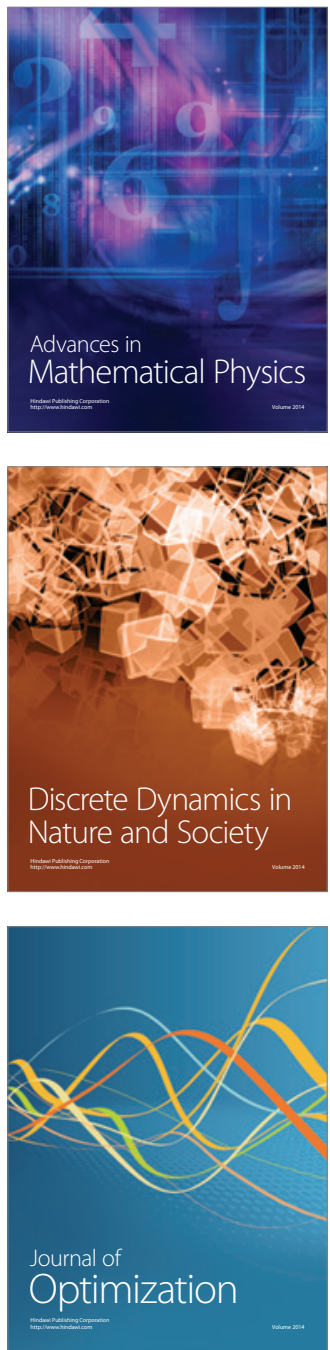